Original paper

\title{
Assessment of clinically relevant dose distributions in pelvic IOERT using Gafchromic EBT3 films
}

\author{
Filipa Costa ${ }^{a}$, Sandra Sarmento ${ }^{\mathrm{a}, \mathrm{b},{ }^{*}, \text { Olga Sousa }}{ }^{\mathrm{c}}$ \\ ${ }^{a}$ Medical Physics, Radiobiology and Radiation Protection Group, Research Centre, Portuguese Institute of Oncology, Porto, Portugal \\ ${ }^{\mathrm{b}}$ Medical Physics Department, Portuguese Institute of Oncology, Porto, Portugal \\ ${ }^{\mathrm{c}}$ Radiation Oncology Department, Portuguese Institute of Oncology, Porto, Portugal
}

\section{A R T I C L E I N F O}

\section{Article history:}

Received 18 March 2015

Received in revised form

6 May 2015

Accepted 20 May 2015

Available online 12 June 2015

\section{Keywords:}

Rectal cancer

Pelvic IOERT

Gafchromic EBT3 films

Dose distributions

\begin{abstract}
A B S T R A C T
Purpose: In IOERT a single dose of radiation is delivered to the tumour site during surgery. Manual dose calculations are used and the irradiation target volume, electron energy and applicator are decided on site by the radiation oncologist. This work assesses the effect that irregular and curved surfaces, typical of pelvic IOERT, may have on the expected dose distribution.

Methods: The feasibility of using Gafchromic EBT3 films and a slab phantom to obtain 2D dose distributions was investigated. Different set-ups were tested by comparison with water tank measurements, applying the gamma function analysis with $2 \%$ and $2 \mathrm{~mm}$ criteria. The validated set-up was then used to obtain reference dose distributions, which were converted to colour-coded graphical representations. Phantoms with step-like and curved surfaces were created to simulate typical pelvic IOERT irradiation surfaces, and the dose distributions were obtained and compared with the reference distributions. Results: Good agreement with water tank measurements was obtained for all applicators below $2 \mathrm{~mm}$, using the chosen setup in reference conditions. In non-reference conditions, the presence of a step-like surface creates an adjacent hotspot, followed by a quick reduction of the dose in depth. With curved surfaces, the dose distribution is shifted forward, becoming curved and deeper, but when the applicator is larger than the hole, hotspots are also observed.

Conclusions: The shape of the irradiation surfaces alters the dose distribution. Visualization of these effects is important to assess target coverage and interpret in vivo measurements in pelvic IOERT.

( $) 2015$ Associazione Italiana di Fisica Medica. Published by Elsevier Ltd. This is an open access article under the CC BY-NC-ND license (http://creativecommons.org/licenses/by-nc-nd/4.0/).
\end{abstract}

\section{Introduction}

The technique of Intra-Operative Electron Radiation Therapy (IOERT) uses ionizing radiation during a surgical intervention, for direct treatment of the tumour site and resection margins, while nearby sensitive tissues are removed from the treatment beam [1-3]. High energy electrons have a relatively short range, compared to high energy photons, thus sparing healthy organs under the target.

Treatment planning for IOERT is usually limited to manual calculations. An alternative was developed through the creation of an IOERT planning software, but this uses a preoperative CT-scan, which does not take into account the alterations to patient

\footnotetext{
* Corresponding author. Medical Physics Department, Portuguese Institute of Oncology, Porto, Portugal.

E-mail address: ssarment@gmail.com (S. Sarmento).
}

anatomy during surgery. Pascau et al. refer the use of an intraoperative CT-scan, but this is also limited by hospital logistics, and not possible in every situation [4]. Without an intraoperative CTscan, the volume to be irradiated is visually estimated in the operating theatre, and the electron energy is chosen by considering the depth of tissue to be treated and the isodose curves measured in reference conditions for that applicator. Dose prescription is usually to the $90 \%$ isodose level, which should include the volume to be treated $[5,6]$. Therefore, it is important to give radiation oncologists a graphical representation of isodoses, to aid them in their visual estimations.

Depending on the situation, further dosimetric studies may be recommended to account for different IOERT scenarios, such as the presence of air gaps [6]. Which effects need to be considered, and how difficult the visual estimation is, depends largely on the anatomical region to be treated. In breast IOERT, for example, nonbevelled applicators are usually sufficient, and it is relatively easy to ensure a flat irradiation surface at the end of the applicator [7-9]. 
Large air gaps may be unavoidable, for example, when treating sarcomas of the lower limb with a movable collimator device $[10,11]$. At our institution, IOERT is mostly used in the pelvic region, to treat advanced and/or recurrent rectal cancer. So bevelled applicators $\left(30^{\circ}\right.$ and specially $\left.45^{\circ}\right)$ are frequently used, and the irradiated surface is usually irregular and/or concave and cannot easily be made flat. Rectal cancer is the second most frequent tumour treated by IOERT in Europe, following breast cancer, so it is essential to ensure the treatment is optimized [12].

In order to characterize an IOERT system, dose profiles are measured in a water phantom at different depths, using a small diode detector, and then the isodose curves are generated by interpolation. However, this process is extremely time consuming $[5,13]$, as profiles have to be acquired for different applicator diameters, bevel angles and electron energies. Moreover, it is difficult to measure the effect of irregular and/or concave irradiation surfaces in a water phantom.

Film dosimetry is generally a good alternative to obtain 2D dose distributions, when compared with point detectors such as ion chambers and diodes. Films have been used to verify field matching for large field irradiations [14] and to study the effect of a beam shaping device [10], using mobile IOERT accelerators. Gafchromic films are suitable to use in IOERT measurements, since they have high sensitivity, low energy dependence and tissue equivalence [15-17]. They can be used either perpendicular or parallel to the incident beam. However, for high energy electrons, obtaining dose distributions with film in the parallel direction can be a challenge, due to the effect of air gaps between film and phantom, which cause artefacts on the dose distribution [18,19]. A good setup is crucial to obtain good results, particularly near the surface where the air gap effect is greater. Nevertheless, some authors have reported good results with films, both perpendicular [20] and parallel to the beam, using conventional [19] and circular [21] electron beam applicators, as well as degraded beams far from the source [22].

The use of films [23,24] (and MOSFETS [7,25]) has also been reported for in vivo IOERT measurements, with good results for relatively flat irradiation surfaces, such as breast, using nonbevelled or $15^{\circ}$ applicators. However, in vivo measurements in pelvic IOERT may be more challenging to execute and interpret, due to the complex irradiation geometries typically found in the pelvic region. Motivation for this work started with attempts to understand the results of in vivo dose measurements in pelvic IOERT, which are currently being performed at our institution.

The aim of this study was to assess the alterations in dose distributions introduced by curved and/or irregular surfaces characteristic of pelvic IOERT, and to determine if such effects are sufficiently relevant to merit consideration, either when interpreting in vivo measurements or for prescription purposes. To this end, we investigated the feasibility of using Gafchromic EBT3 films and a solid water phantom to obtain complete dose distributions parallel to the beam, for IOERT applicators of different diameters and bevel angles. These IOERT dose distributions were compared with previous measurements in water tank, to validate the methodology. The validated irradiation setup was then used to study the effects of typical pelvic IOERT surface irregularities on dose distributions, using phantoms created in-house. Surface dose was also assessed to compare with in-vivo measurements.

\section{Materials and methods}

\section{LINAC and IOERT system}

IOERT procedures at our institution are performed with a Varian Clinac 2100 CD conventional linear accelerator (LINAC), located adjacent to an operating theatre, and adapted for IOERT with a hard docking system of cylindrical applicators shown in Fig. 1A. All measurements reported in this work were performed using this LINAC, and either a conventional $10 \times 10 \mathrm{~cm}^{2}$ electron applicator, or the IOERT applicators most frequently used clinically, which are 6, 7 and $8 \mathrm{~cm}$ in diameter, with bevel angles of $0^{\circ}$ (B0), $30^{\circ}$ (B30) and $45^{\circ}$ (B45). The study was restricted to those electron energies typically used in IOERT treatments (6, 9 and $12 \mathrm{MeV}$ ), specially $9 \mathrm{MeV}$ which is used most frequently at our institution for pelvic IOERT. The sourceto-surface distance (SSD) of $134 \mathrm{~cm}$, which is the standard SSD for this IOERT applicator system, was used for all measurements.

Percentage depth dose (PDD) curves and dose profiles had previously been acquired in a water phantom (MP3-M, PTWFreiburg, Germany), using a diode (type 60012 E PTW-Freiburg, Germany), for the available electron beam energies and for IOERT and conventional $10 \times 10 \mathrm{~cm}^{2}$ electron beam applicators. As recommended by AAPM task group 48, with IOERT applicators the PDDs were obtained along the "clinical axis", which is defined by a line perpendicular to the phantom surface and intersecting the central axis of the applicator at the surface [2], as shown in Fig. 1A. For simplicity, this is referred in the text as central axis. Absorbed dose to water was measured with either a Roos (type 34001, PTWFreiburg, Germany) or Markus (type 23343, PTW-Freiburg, Germany) ionization chamber, according to IAEA TRS 398 recommendations [26]. These measurements were part of the initial characterization of the LINAC and the IOERT system.

\section{Gafchromic EBT3 films}

For this work, irradiations were performed using Gafchromic EBT3 film (International Speciality Products, Wayne, NJ, USA) and a white polystyrene slab phantom (type RW3 PTW-Freiburg, Germany) to obtain dose profiles. This solid water phantom, usually referred as a water-equivalent material, was used as an alternative to water tank following the recommendations of IAEA TRS 398 $[26,27]$. Gafchromic EBT3 films were taped to a phantom slab and irradiated parallel to the beam in order to obtain IOERT and conventional $10 \times 10 \mathrm{~cm}^{2}$ electron beam profiles.

An Epson expression 10000XL was used to scan the Gafchromic EBT3 films, 23 and $48 \mathrm{~h}$ after irradiation. Each film was placed on the centre of the scanner to avoid scanner-induced nonuniformity and digitized in landscape orientation and transmission mode. The digitized images were analysed using the Image J software (National Institute of Health, Bethesda, MA, USA) and the data were extracted from the red colour channel, which the manufacturer indicates as more advantageous below $10 \mathrm{~Gy}$. Triple channel analysis was not used for these phantom measurements [28].

A third degree polynomial calibration curve was obtained to convert optical density (OD) to dose, for doses ranging from 0 to 18 Gy. The mean pixel value (MPV) of each film was first determined and then converted to OD. Eleven pieces of film of $5 \times 5 \mathrm{~cm}^{2}$ were irradiated using a non-bevelled $8 \mathrm{~cm}$ applicator and a $9 \mathrm{MeV}$ electron beam, while another piece of film was left unexposed for background correction. Initial calibrations were obtained for two sets of films, one placed at the surface of the phantom and the other at the depth of dose maximum $\left(\mathrm{d}_{\max }\right)$. Subsequent calibrations were performed only at the surface, since the conversion factor was already known. Film calibration was repeated every $4-5$ months to account for the autodevelopment of films, which tend to darken even in the absence of radiation [29].

\section{Image analysis}

PDD curves and dose profiles were obtained from the parallel irradiated films, using the plot profile function from ImageJ software. After background correction, the calibration curve was 

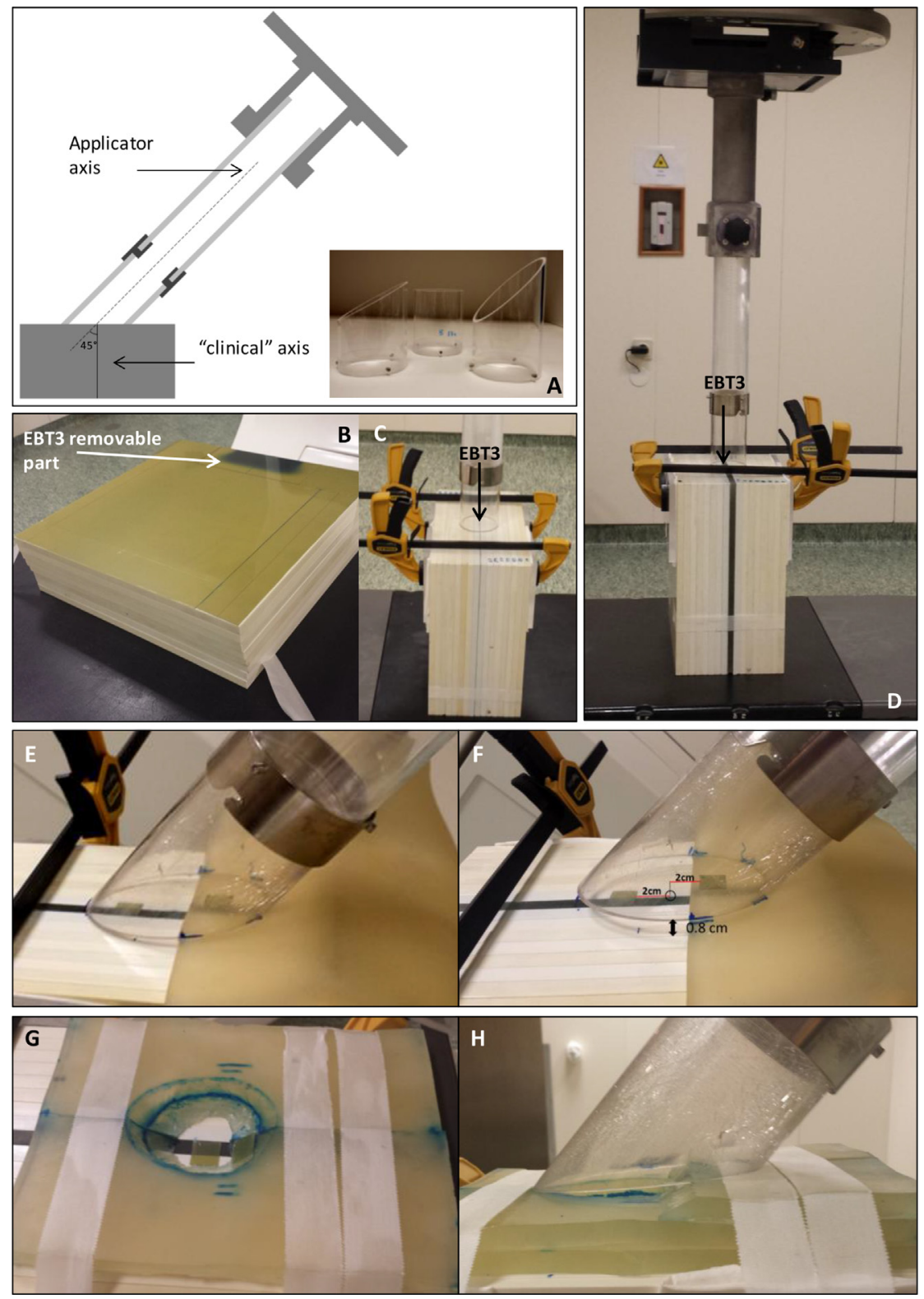

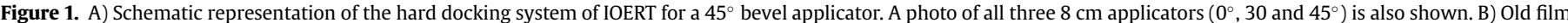

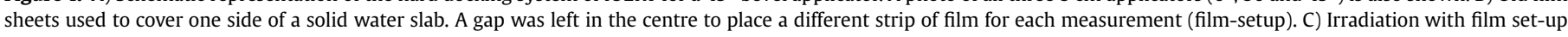

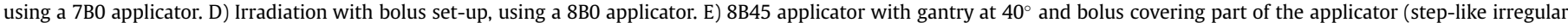

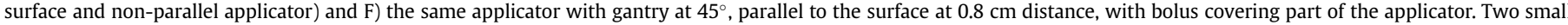

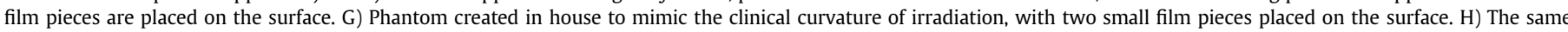
phantom with an additional cut bolus and 8B45 applicator and gantry at $40^{\circ}$.

applied to convert OD to dose. The results of this analysis were compared with PDD curves and profiles obtained in a water tank. Analysis of profiles was initially performed based on visual assessment. For a more quantitative comparison, gamma function analysis with $2 \mathrm{~mm}$ distance to agreement (DTA) and 2\% dose difference criteria was applied [30].

Depending on the beam energy, some water tank measurements with the diode were obtained at spatial intervals of $1 \mathrm{~mm}$ or more, particularly at greater depths, while the Gafchromic EBT3 film was scanned with 72 dots per inch (DPI), resulting in a value of dose each $0.35 \mathrm{~mm}$. The sampling differences of each measurement made it impossible to obtain good results using the gamma function, even when a perfect match between the curves was visualized. Therefore, before applying the gamma analysis, it was necessary to interpolate linearly the diode measurements in order to obtain results at spatial intervals comparable to the film (steps of $0.5 \mathrm{~mm}$ ). These interpolated points will not be displayed graphically in the figures shown in the results section.

2D dose distributions were obtained from the digitized images using Matlab, for further comparison with different irradiation scenarios created in house.

Furthermore, to confirm that the calibration curve (obtained with films perpendicular to the beam) could be applied to films irradiated in the parallel direction with sufficient accuracy for normalization purposes, the central axis OD at $\mathrm{d}_{\max }$, obtained from films parallel to the beam was converted to dose $\left(D_{\max }\right)$, and compared with the central axis maximum dose calculated from water tank measurements. 
Slab phantom - IOERT reference conditions

The methodology proposed by Barouky et al. was adapted for the purposes of this work to avoid the effect of air gaps when using Gafchromic film parallel to the beam in a slab phantom. Originally, Barouky et al. used clamps to apply pressure, combined with ultrasound (US) gel and 2 layers of film on the surface of the phantom [19]. In this work, the US gel and the two extra film pieces could not be used. Due to limited machine time, several measurements had to be performed quickly while keeping the surrounding area clean, therefore the use of gel would have been inconvenient. Concave irradiation surfaces are also incompatible with two extra films covering the phantom. Therefore, a suitable alternative was developed.

Gafchromic EBT3 films were cut with the essential dimensions to comprise the entire profile, depending on the applicator, bevel and energy used. The films were then sandwiched between 16 solid phantom slabs. We observed that even the slightest curvature of the slabs increases the air gap effect. To reduce this issue, phantom slabs were numbered and used always in the same order. Pressure was applied on both sides of the phantom, using clamps and $12 \mathrm{~cm}$ square slabs of acrylic to distribute the pressure evenly and prevent mechanical damage to the phantom slabs (Fig. 1C and D). Although film height never exceeded $6 \mathrm{~cm}$, as an additional precaution to avoid any bowing effect, adhesive tape was used on the lower part of the phantom for all measurements.

Two different set-ups were investigated. For the first set-up (film set-up), one of the middle slabs was covered with old film, fixed in place with adhesive tape, leaving only a small uncovered space at the top, where a new strip of film could be inserted for each measurement. This smaller piece also had to be held in place with adhesive tape. This setup creates a flat surface for a more uniform connection to the adjacent slab (Fig. 1B and C). The other set-up (bolus set-up) consisted in sandwiching a $1 \mathrm{~cm}$ thick $\left(30 \times 30 \mathrm{~cm}^{2}\right)$ radiotherapy bolus (Superflab, Mick Radio-Nuclear Instruments) between the 16 solid water slabs, and placing a small piece of film between the bolus and the adjacent slab (Fig. 1D). The elasticity of the bolus ensures good adaptation to adjacent surfaces, without air gaps. Care was taken to limit the applied pressure, so as to avoid as much as possible the bulging effect which tends to occur as a result of this elasticity.

Due to limited availability of IOERT applicators, initial measurements were performed with a conventional $10 \times 10 \mathrm{~cm}^{2}$ electron beam applicator, in order to test the methodology and compare the two set-ups. Data were then obtained for the $7 \mathrm{~cm}$ applicator, for the three bevel angles and 6 and $9 \mathrm{MeV}$, using film set-up (Fig. 1C) and bolus set-up (Fig. 1D), because the effects could be different using the cylindrical applicators. The bolus set-up was then used to obtain data for all other applicators $(6$ and $8 \mathrm{~cm}$, with bevel angles of $0^{\circ}, 30^{\circ}$ and $45^{\circ}$ for 6,9 and $12 \mathrm{MeV}$ ) in IOERT reference conditions. For the purposes of this work, IOERT reference conditions refers to an irradiation geometry comparable to water tank measurements, meaning a flat irradiation surface with the applicator parallel to it and in contact with the phantom.

Each film was irradiated with 500 Monitor Units (500 MUs). The centre of the phantom was aligned with the beam axis and the corresponding position in the film was marked.

\section{Slab phantom - irregular irradiation surfaces}

The bolus set-up shown in Fig. 1D was adapted to simulate different irradiation scenarios, combining surface irregularities and partial air gaps (where the bevelled end of the applicator is not parallel to the surface to be irradiated). In pelvic IOERT, the irradiation surface is often concave, as a result of the sacrum curvature.
Sometimes there is also tissue partially covering the applicator opening, usually when the applicator does not fit perfectly in both width and length (the choice is limited by available sizes). As a result of this complex geometry, it is often not possible to place the applicator parallel to the surface. Therefore, to study the influence of non-parallel irradiation, the bevelled applicator was placed at a $5^{\circ}$ inclination (Gantry at $40^{\circ}$ rather than $45^{\circ}$ ) relative to the standard position leaving an air gap between the bevelled end and the phantom. The couch position was adjusted to centre the phantom. To simulate tissue partially covering the applicator opening, a piece of radiotherapy bolus was used, creating a step-like irradiation surface as shown in Fig. 1E. To allow separate comparison of the influence of each parameter, an intermediate situation was considered, consisting of a step-like surface with the bevelled applicator parallel to the phantom surface, at $0.8 \mathrm{~cm}$ distance, allowing the malleable bolus to be positioned partly in between, as shown in Fig. 1F.

For more realistic clinical scenarios, two other pieces of bolus were used to create a phantom with a curved surface: these pieces were cut in half to allow positioning of the Gafchromic EBT3 film between them. A concave hole was dug to create a curvature with the same inclination as an average male sacral bone (Fig. 1G and H). The curvature was determined based on the average dimensions measured on DICOM CT images from 7 male IOERT patients from our institution. The film was also cut to match the irregular surface created with the bolus. The effect of this curved surface with approximately $2 \mathrm{~cm}$ depth (Fig. 1G) was studied using 6 and $8 \mathrm{~cm}$ applicators with $45^{\circ}$ bevel parallel to the surface (gantry at $45^{\circ}$ ). An additional piece of bolus was placed on one side of the phantom to increase the inclination of the ramp, and the effect studied using the $8 \mathrm{~B} 45$ applicator positioned non-parallel to the surface (gantry at $40^{\circ}$ ), as shown in Fig. $1 \mathrm{H}$. All films were irradiated with 500 MUs and a $9 \mathrm{MeV}$ electron beam. To assemble these phantoms two persons are needed. The bolus material is slightly adhesive, which helps prevent air gaps and keeps the cut Gafchromic film aligned with the first half bolus (simulating the sacral bone curvature), while this is aligned with the bolus in the middle of the solid water phantom. The remaining water phantom slabs are added next and held in place with the clamps. The other half of the cut bolus is added last, and pressure applied to eliminate air gaps, first manually and then with adhesive tape (Fig. $1 \mathrm{G}$ and H).

\section{Surface measurements}

For comparison with in vivo measurements currently being done at our institution, some surface measurements were performed in non-reference situations, using small pieces of EBT3 film $\left(1.5 \times 1.5 \mathrm{~cm}^{2}\right)$, placed on the top of the phantom. For the three irradiation scenarios which do not involve curved surfaces, 2 pieces of film were placed $2 \mathrm{~cm}$ away from the beam central axis (Fig. 1E and F). For the curved surfaces shown in Fig. $1 \mathrm{G}$ and $\mathrm{H}$, a film piece was placed at the centre and another on the slope for the three different irradiations scenarios. The mean dose distribution was calculated within each film piece.

In reference situations we have a flat surface and the applicator is parallel and touching the phantom, which is very different from a clinical irradiation scenario. One sheet of Gafchromic film was placed at the surface of 12 horizontal phantom slabs and another at $\mathrm{d}_{\text {max }}$, for all the applicators $(6,7$ and $8 \mathrm{~cm})$ and the three available bevel angles. Irradiations were performed with $9 \mathrm{MeV}$ electron beam and 500 MUs. The irradiated film sheets were digitized, and surface profiles were obtained from a central rectangular region of interest (ROI) of $1.5 \mathrm{~cm}$ width, to compare with the surface measurements performed in non-reference situations (Fig. 1E-H), with the $1.5 \times 1.5 \mathrm{~cm}^{2}$ film pieces. 


\section{Results}

\section{Preliminary measurements with a conventional applicator}

PDD curves were obtained with three different set-up configurations, for a $10 \times 10 \mathrm{~cm}^{2}$ conventional electron applicator (Fig. 2). Previous water tank measurements are included in Fig. 2 for comparison, represented as points to indicate measuring positions. Film results are represented as lines.

Using only a piece of Gafchromic EBT3 film in the middle of the solid slabs resulted in poor agreement between the PDD curve obtained with film and the one acquired in a water tank phantom, as previously reported by Barouky et al. [19]. This method requires fixing the film in place with tape, which may create an additional air gap, particularly when small pieces of film are used. Applying the gamma function, differences are especially noticeable near the surface and good agreement (gamma $<1$ with $2 \%, 2 \mathrm{~mm}$ criteria) was obtained only below $3.9 \mathrm{~mm}$ depth.

The two different set-ups shown in Fig. 2 (bolus set-up and film set-up) were investigated next. As shown in Fig. 2, the film set-up gives good agreement if we exclude the initial measurement points where $\approx 3 \%$ underdose is observed due to air gaps that could not be eliminated. Applying the gamma function, for a more quantitative assessment, a good agreement is achieved beyond $1.8 \mathrm{~mm}$. The bolus set-up produced good results over the entire curve, with maximum $1 \%$ difference when compared with the diode data. This is confirmed by the gamma function where the gamma value is less than 1 throughout the entire PDD curve (with $2 \%, 2 \mathrm{~mm}$ criteria).

Measurements with IOERT applicators in standard conditions and validation of methodology

PDD curves obtained with both film set-up (Fig. 1C) and bolus set-up (Fig. 1D), for the $7 \mathrm{~cm}$ applicator (bevel $0^{\circ}, 30^{\circ}$ and $45^{\circ}$ ) with 6 and $9 \mathrm{MeV}$, agree well with the water tank measurements. Artefacts are sometimes observed in the first pixels of the digitized images, and had to be removed, particularly for the film set-up, which also proved to be time consuming and impractical. Therefore, measurements for the other IOERT applicators were performed using only the bolus set-up, which was easier and faster to position.

PDD curves obtained for the $8 \mathrm{~cm}$ diameter applicator with bevel angles of $0^{\circ}, 30^{\circ}$ and $45^{\circ}$, for $9 \mathrm{MeV}$ electron beams, are shown in Fig. 3 and compared with the PDD curves obtained in water tank. The results were compared by visual assessment and gamma function analysis with $2 \%$ and $2 \mathrm{~mm}$ criteria, shown in the secondary axes of Fig. 3. For the $8 \mathrm{~B} 0$ applicator, the first $0.7 \mathrm{~mm}$ had

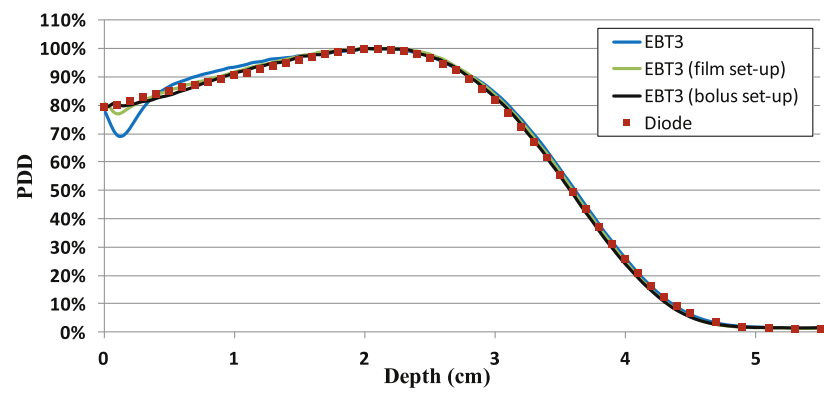

Figure 2. PDD curves obtained with Gafchromic EBT3 film and a solid water phantom, using three different irradiation set-ups. EBT3 (film piece sandwiched between phantom slabs), EBT3 bolus set-up and EBT3 film set-up. These results were compared with the PDD curve obtained with a diode in a water tank.
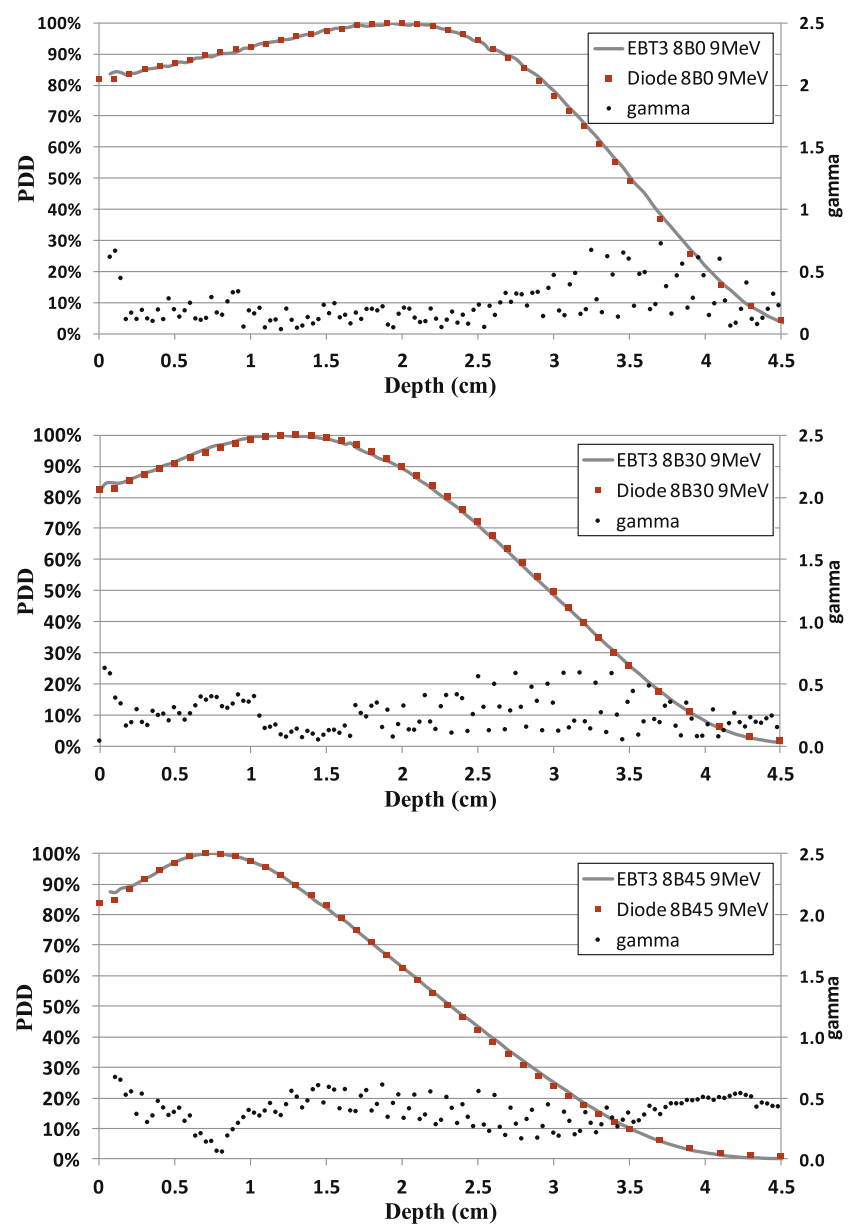

Figure 3. PDD curves obtained with bolus set-up, using three different bevel angles A) B0, B) B30 and C) B45 for a $8 \mathrm{~cm}$ applicator and $9 \mathrm{MeV}$ electron beam energy. Gamma values shown in the secondary axes were calculated with a $2 \%$ and $2 \mathrm{~mm}$ criteria.

to be removed due to artefacts, probably resulting from the film cutting process. A good match was found throughout the PDD curves for the $8 \mathrm{~B} 30$. For the $8 \mathrm{~B} 45$, the PDD curves had to be shifted by $0.7 \mathrm{~mm}$.

The films were manually aligned with the top of the phantom, so minor positioning errors may have occurred. Moreover, the bolus has a tendency to curve slightly upwards when compressed. This may explain why some PDD curves have to be shifted by $0.4-0.7 \mathrm{~mm}$, in order to obtain best agreement with water tank measurements. Occasionally, the first $1-2$ pixels $(0.4-0.7 \mathrm{~mm})$ have to be removed due to the presence of artefacts resulting from cutting the film. Other effects were sometimes present, and good agreement was not always obtained in the first $2 \mathrm{~mm}$ of the PDD curves, especially for non-bevelled applicators and smaller diameters ( $6 \mathrm{~cm}$ applicator). Good agreement was obtained between $2 \mathrm{~mm}$ below the surface and the depth where the dose is $10 \%$ of $D_{\max }(R 10)$, for all the applicators, bevels and energies (100\% points with gamma $<1$ with $2 \%$ and $2 \mathrm{~mm}$ criteria).

PDD curves obtained for $8 \mathrm{~B} 45$ for 6 and $12 \mathrm{MeV}$ are shown Fig. 4. The effect of beam energy is clearly visible. PDD curves obtained with $45^{\circ}$ bevel applicators showed particularly good agreement with water tank measurements, when compared with lower bevel angles. This is fortunate, since $45^{\circ}$ bevel applicators are the most clinically used in pelvic IOERT and therefore the main focus of this work. All measurements for 6 and $9 \mathrm{MeV}$ with $45^{\circ}$ bevel applicators $(6,7$ and $8 \mathrm{~cm})$ would have passed the gamma function tests with a 

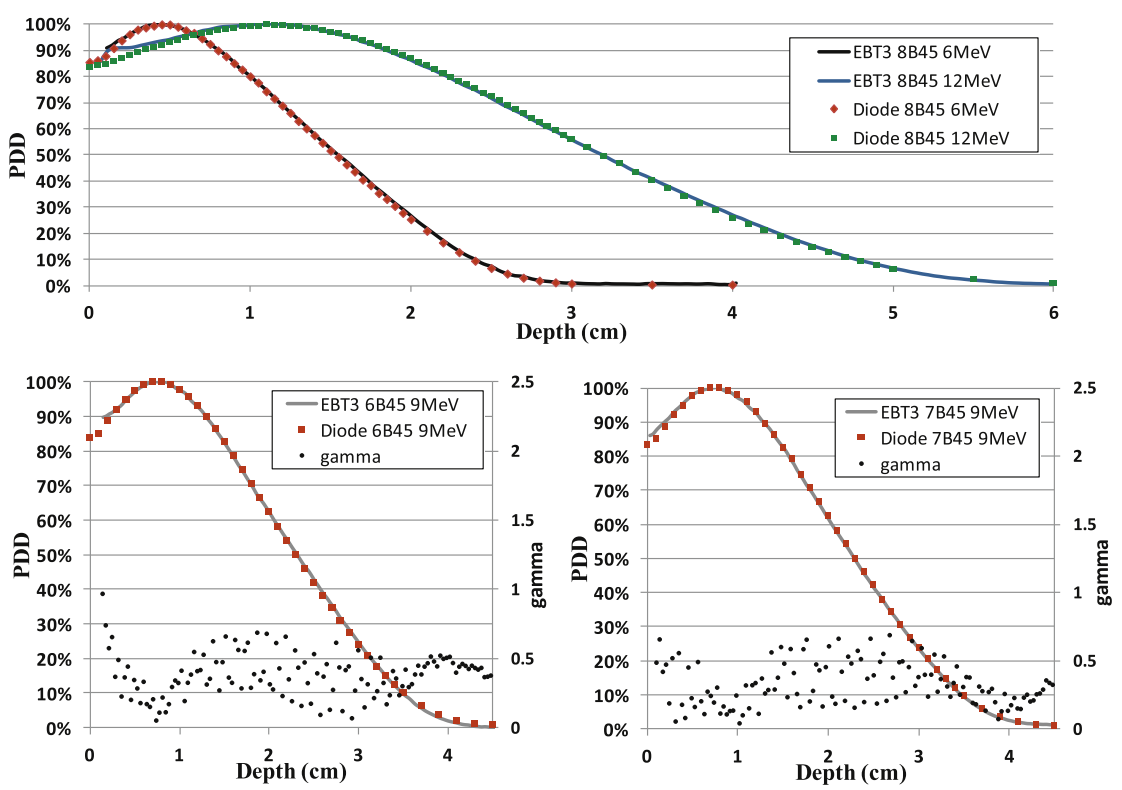

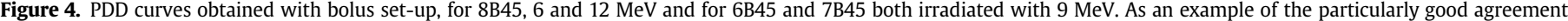
found for $45^{\circ}$ applicators, gamma values obtained with a tighter criteria ( $1.5 \%$ and $1.5 \mathrm{~mm}$ ) are displayed in the secondary axis for $6 \mathrm{~B} 45$ and $7 \mathrm{~B} 45$ applicators.

tighter criteria of $1.5 \%$ and $1.5 \mathrm{~mm}$, between $2 \mathrm{~mm}$ and R10. Two examples of this are shown in Fig. 4, for applicators 6 and $7 \mathrm{~cm}$ with a $45^{\circ}$ bevel angle and $9 \mathrm{MeV}$ electron beams, where the gamma function with $1.5 \%$ and $1.5 \mathrm{~mm}$ criteria is displayed on the secondary axes of the graphs. However, for consistency, we decided to consider only the gamma criteria of $2 \%$ and $2 \mathrm{~mm}$ for all applicators.

Good agreement was also obtained for lateral dose profiles. Examples of the dose profiles obtained at different depths, including $d_{\max }$, are shown in Fig. 5 for the three IOERT applicators and $9 \mathrm{MeV}$ electron beam.

The digitized films which were converted to 2D distributions are shown in Fig. 6, for the three different bevels angles of an $8 \mathrm{~cm}$ applicator and $9 \mathrm{MeV}$. A colourwash was used to distinguish isodoses. The shape of the bevels is drawn in scale, for a better perception of the dose distribution in relation to the bevel of the applicator. The 2D dose distribution for a 6B45 applicator and $9 \mathrm{MeV}$ electron beam is also displayed in the same figure.

The dose distributions were normalized to the mean maximum dose of a selected ROI on the central axis. This procedure is analogous to the habitual practice of normalizing PDD curves and profiles to the maximum central axis dose, in water tank measurements. The images colourmap is displayed in steps of $5 \%$ from $0 \%$ to $110 \%$, with isodose lines in steps of $10 \%$, and where $100 \%$ corresponds to the value of maximum dose at the central axis. The images are displayed until $110 \%$ for further comparison with the isodoses obtained in non-reference conditions.

In Table 1 the central axis maximum doses $\left(D_{\max }\right)$ obtained from films are compared with the expected values calculated from tables and water tank measurements. The differences observed are in the range of $+0.5 \%$ to $-3 \%$.

\section{Measurements with IOERT applicators and irregular surfaces}

The dose distributions obtained from the irradiation with nonparallel 8B45 applicator (gantry at $40^{\circ}$ ), and the step-like surfaces shown in Fig. 1E and F, are presented in Fig. 7. The results can be compared with the reference distribution presented in Fig. 6, for a 8B45 applicator. The non-standard distributions of Fig. 7 were normalized to the value of dose maximum for the standard distribution. Therefore, $100 \%$ in Fig. 7 corresponds to the same value of dose as $100 \%$ in Fig. 6 for $8 \mathrm{~B} 45$ and $9 \mathrm{MeV}$.

For a quantitative analysis of the non-reference situation, and to obtain PDD curves comparable to the reference, three ROIs were selected. One central ROI (centre_ROI) and two others, one on the
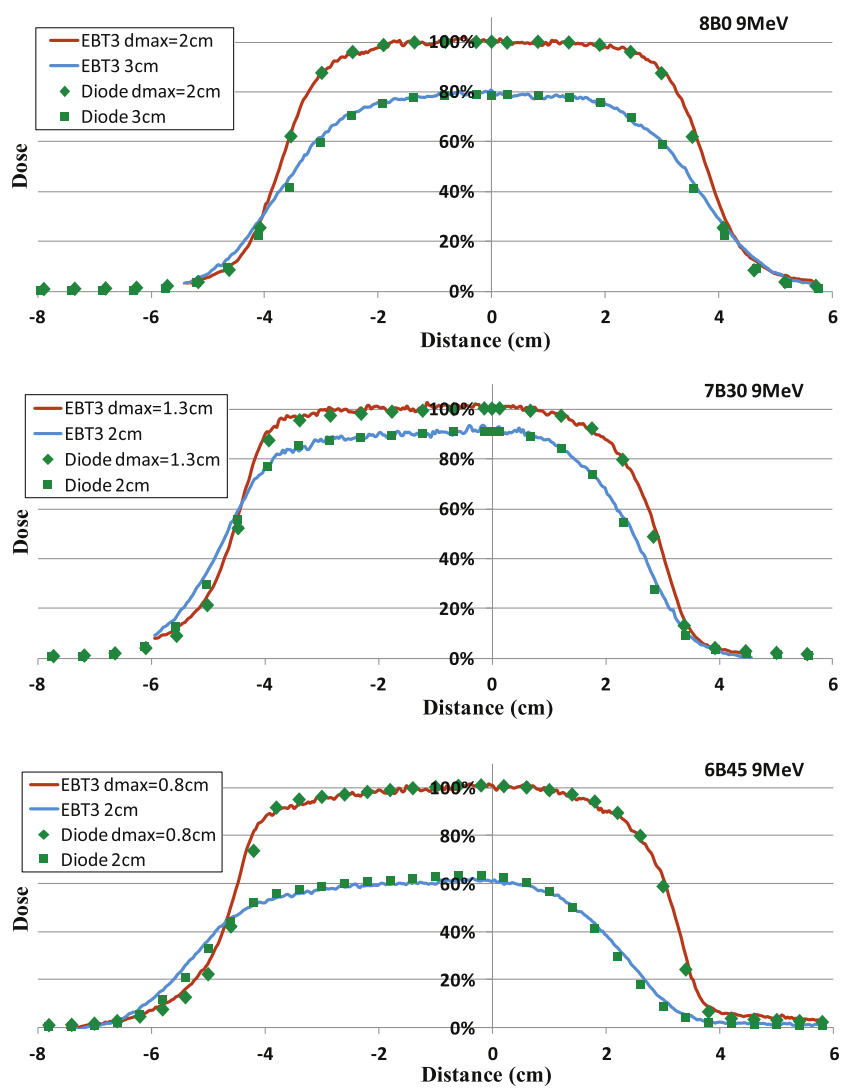

Figure 5. Lateral depth dose profiles obtained with Gafchromic EBT3 film using the bolus set-up and with diode in a water tank for $\mathrm{d}_{\max }$ and other value of depth, for 8,7 and $6 \mathrm{~cm}$ applicators, with bevel angles of $0^{\circ}, 30^{\circ}$ and $45^{\circ}$ respectively. 

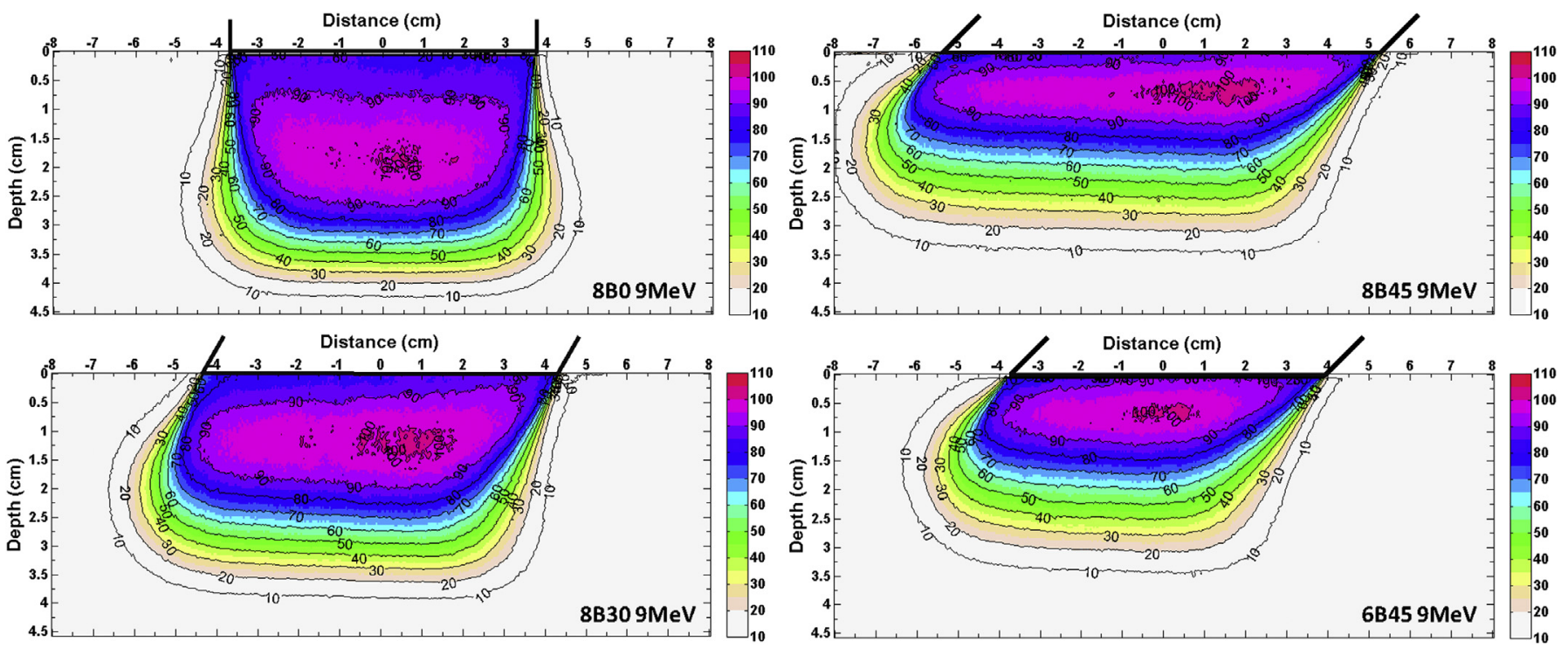

Figure 6. Dose distribution for 8B0, 8B30, 8B45 and 6B45 applicators obtained with film and bolus set-up, and irradiated with $9 \mathrm{MeV}$ electron beam, for comparison with non-flat surfaces and/or non-parallel irradiation. The applicators and their positions are outlined above. The isodose curves for $8 \mathrm{~B} 0$ are not symmetrical. This asymmetry is inherent to the applicator system. It is also visible in the lateral profiles obtained in water tank (Fig. 5), and in the results published by José del Río et al. [5], for a similar IOERT system.

longer part of the applicator (left_ROI) and another on the shorter part of the applicator (right_ROI). The PDD curves for each selected ROI for one of the step-like surfaces (Fig. 7C) are shown in Fig. 8. For the right_ROI a shift in depth of $0.8 \mathrm{~cm}$ was applied, to account for the extra bolus tissue placed on the phantom surface. The PDD curves obtained in the same ROIs for the reference situation (8B45 of Fig. 6) are also displayed for comparison.

The dose distributions obtained with curved irradiation surfaces are shown in Fig. 9A-C. The results obtained for the irradiation surface shown in Fig. 1G, with applicators 6B45 and 8B45 are presented in Fig. 9A and B, respectively. The dose distribution obtained with the set up displayed on Fig. $1 \mathrm{H}$ is presented in Fig. 9C. The applicators and their respective positions are outlined. The surface artefacts from cutting the Gafchromic film are clearly visible close to the surface in all 2D dose distributions presented in Fig. 9, as they could not be removed.

\section{Surface measurements}

The surface dose profile obtained for the 8B45 applicator and $9 \mathrm{MeV}$, in reference conditions, is presented in Fig. 10. Mean values of dose obtained from the $1.5 \times 1.5 \mathrm{~cm}^{2}$ film pieces in non-reference conditions are also shown in the same graph, for comparison.

\section{Table 1}

Roos ionization chamber and Gafchromic EBT3 film readings at $\mathrm{d}_{\max }$ based on irradiation performed in a water phantom and with bolus set-up, respectively, both irradiated with $9 \mathrm{MeV}$ electron beam. Readings of the detectors were corrected for the influence quantities of temperature and pressure.

\begin{tabular}{lcllr}
\hline Applicator $(\mathrm{cm})$ & Bevel $\left({ }^{\circ}\right)$ & $D_{\max } \operatorname{Roos}(\mathrm{Gy})$ & $\mathrm{D}_{\max }$ EBT3 $(\mathrm{Gy})$ & Diff $(\%)$ \\
\hline $10 \times 10$ & - & 5.07 & 5.10 & $0.5 \%$ \\
6 & 0 & 3.16 & 3.13 & $-1.1 \%$ \\
& 30 & 3.32 & 3.35 & $1.0 \%$ \\
7 & 45 & 3.43 & 3.38 & $-1.6 \%$ \\
& 0 & 3.49 & 3.44 & $-1.3 \%$ \\
& 30 & 3.69 & 3.63 & $-1.6 \%$ \\
8 & 45 & 3.75 & 3.66 & $-2.3 \%$ \\
& 0 & 3.69 & 3.59 & $-1.7 \%$ \\
& 30 & 3.90 & 3.86 & $-3.0 \%$ \\
\hline
\end{tabular}

As shown in Fig. 10, most surface readings agree well with the expected reference values, specially for the irradiation geometry shown in Fig. 7C (8B45 step-like surface, parallel alignment). The gantry at $40^{\circ}$, rather than $45^{\circ}$, decreases the surface dose slightly on the right side, for the irradiation geometries shown in Fig. 7A and B. The only values that are markedly different from the reference situation are the ones obtained at the isocentre for the $8 \mathrm{~B} 45$ applicator and curved irradiation surfaces (Fig. 9B and C). For the 6B45 applicator (Fig. 9A), which is not shown in Fig. 10, the measured value at the isocentre was $5 \%$ less than expected for the reference distribution. This was expected from the observation of Fig. 9A, where the dose distribution near the surface, and particularly close to the central axis, seems lower than the reference for the same location (Fig. 6).

\section{Discussion}

The initial PDD curve presented in Fig. 2 (EBT3) shows that a good set-up is crucial to obtain reliable results for high energy electron beams, when using films and solid phantoms parallel to the beam, as reported by Barouky et al. [19]. Both set-ups tested in this study produce reliable dose distributions with Gafchromic EBT3 films placed parallel to the beam, using either a conventional $10 \times 10 \mathrm{~cm}^{2}$ applicator or IOERT applicators. The bolus set-up proved easier and faster to position, with better results than the film set-up, despite the tendency of the bolus to curve slightly upwards when compressed. This may explain why some PDD curves obtained with the bolus set-up needed to be shifted by $0.4-0.7 \mathrm{~mm}$, for best concordance with water tank measurements.

However, despite artefacts and positional uncertainty, good agreement $(100 \%$ points with gamma $<1$ ) was obtained between the PDD curves acquired with the bolus set-up and the ones obtained in water tank, for all applicators, bevels and energies studied, between $2 \mathrm{~mm}$ and R10 (the depth where the dose is $10 \%$ of $\mathrm{D}_{\max }$ ), using gamma function analysis with $2 \%$ and $2 \mathrm{~mm}$ criteria.

The values of $D_{\max }$, obtained from films and summarized in Table 1 , are within $+0.5 \%$ to $-3 \%$ of the expected values calculated from tables. The output of the LINAC was not measured on the day when films were irradiated, so normal output fluctuations were not 

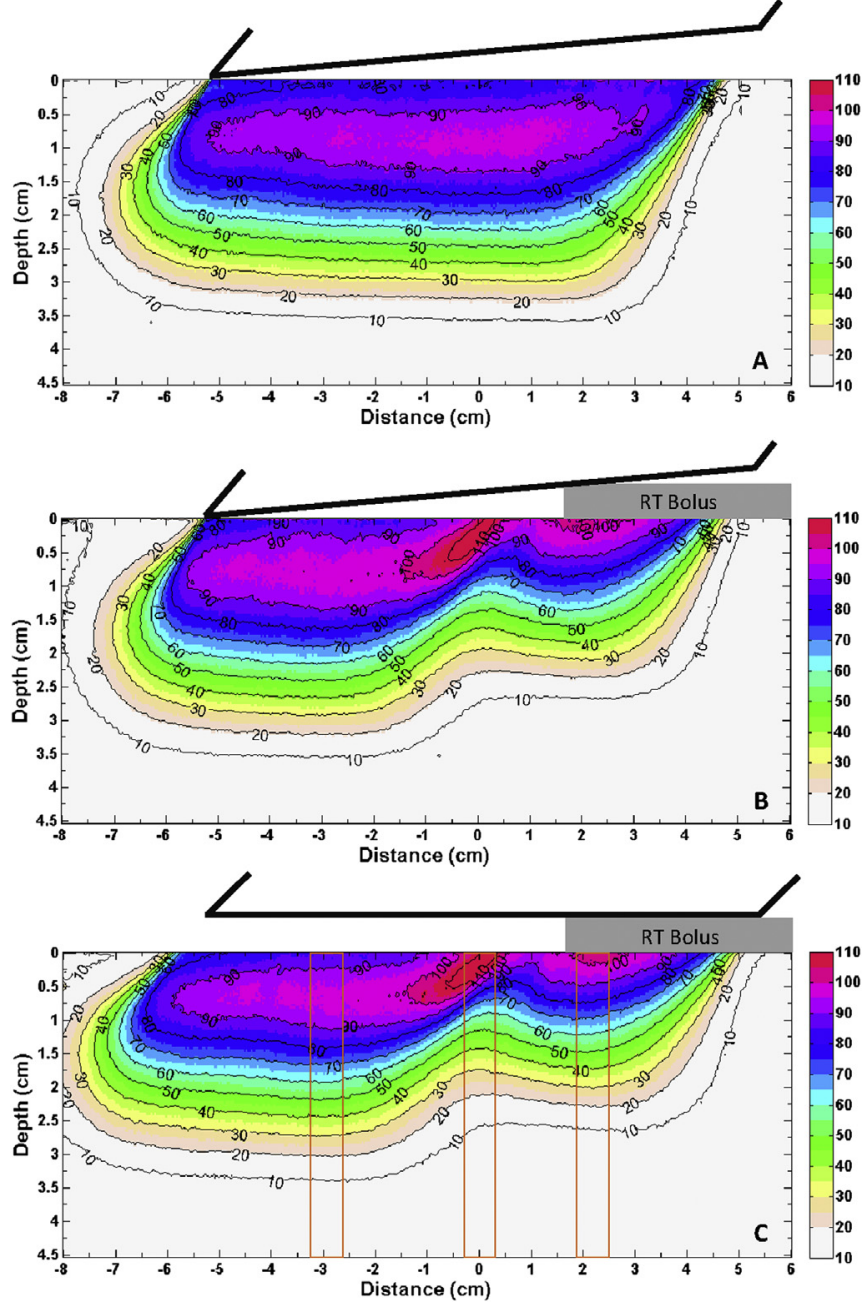

Figure 7. Dose distribution results obtained with Gafchromic film for the three configurations tested. Set-up created for dose distribution B and C are shown in Fig. 1E and F respectively. Dose distributions were normalized to the $D_{\max }$ of the $8 \mathrm{~B} 45,9 \mathrm{MeV}$ reference measurement. The applicator and bolus position is displayed as well as the ROI selected to obtain the PDD curves displayed on Fig. 8. The applicator and bolus position are outlined above.

accounted for. The expected uncertainty of Gafchromic film measurements in optimal conditions and used perpendicular to the beam is $\pm 2 \%[29,31]$, but in this case, the films were irradiated parallel to the beam, and several known sources of uncertainty
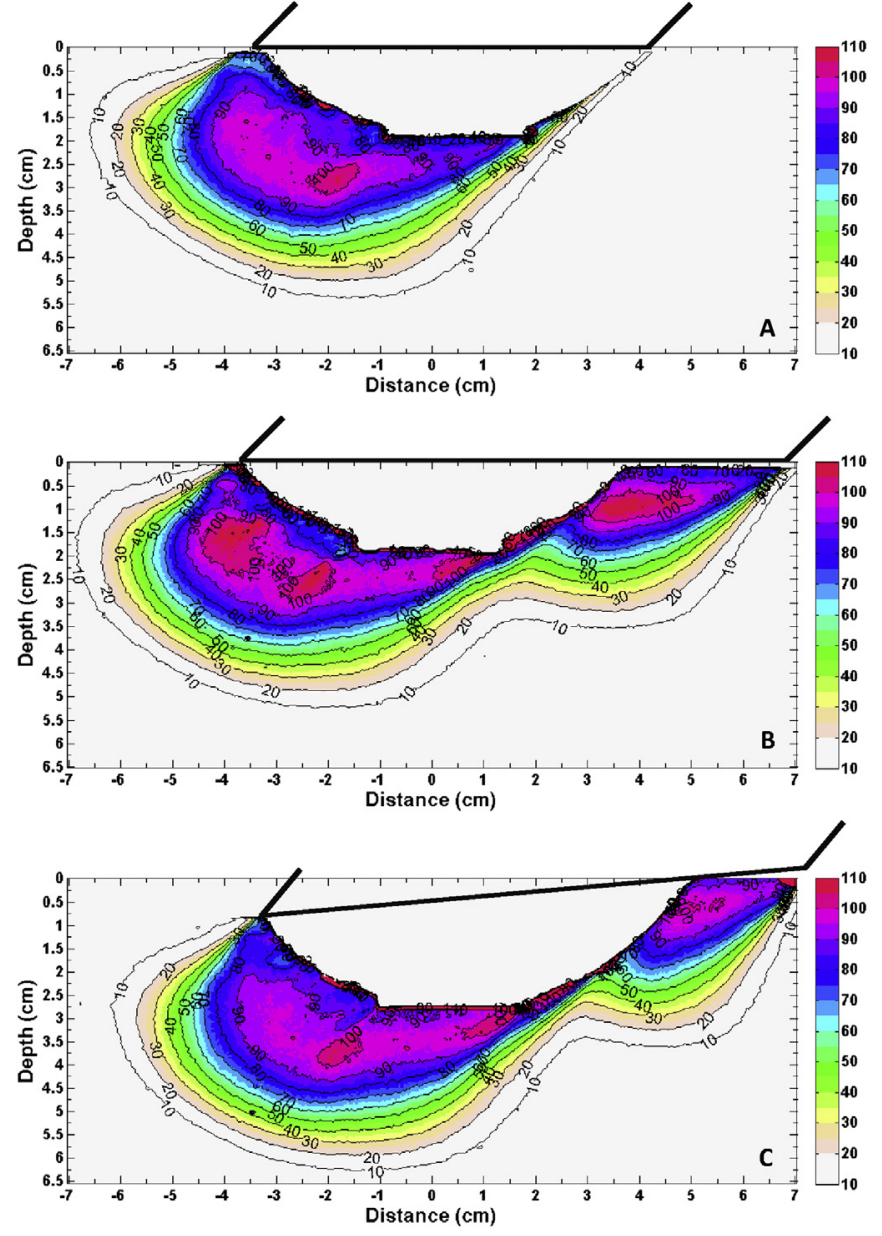

Figure 9. Dose distribution results obtained with Gafchromic film for the two configurations tested with the bolus phantom simulating a hole displayed on Fig. 1G, irradiated with A) 6 B45 and B) 8B45 applicators. The result obtained with the set-up of Fig. $1 \mathrm{H}$ and a $8 \mathrm{~B} 45$ applicator is shown in $\mathrm{C}$ ). The applicator and bolus position are outlined above.

(such as film response non-uniformity, storage temperature and humidity, and temperature variations during the scanning process) could not be controlled. Autodevelopment of the film is another important factor, and differences of $4 \%$ for calibration curves obtained 3 months apart have been reported [29]. Moreover, the PDD curves were obtained from a thin region of interest (ROI), so the
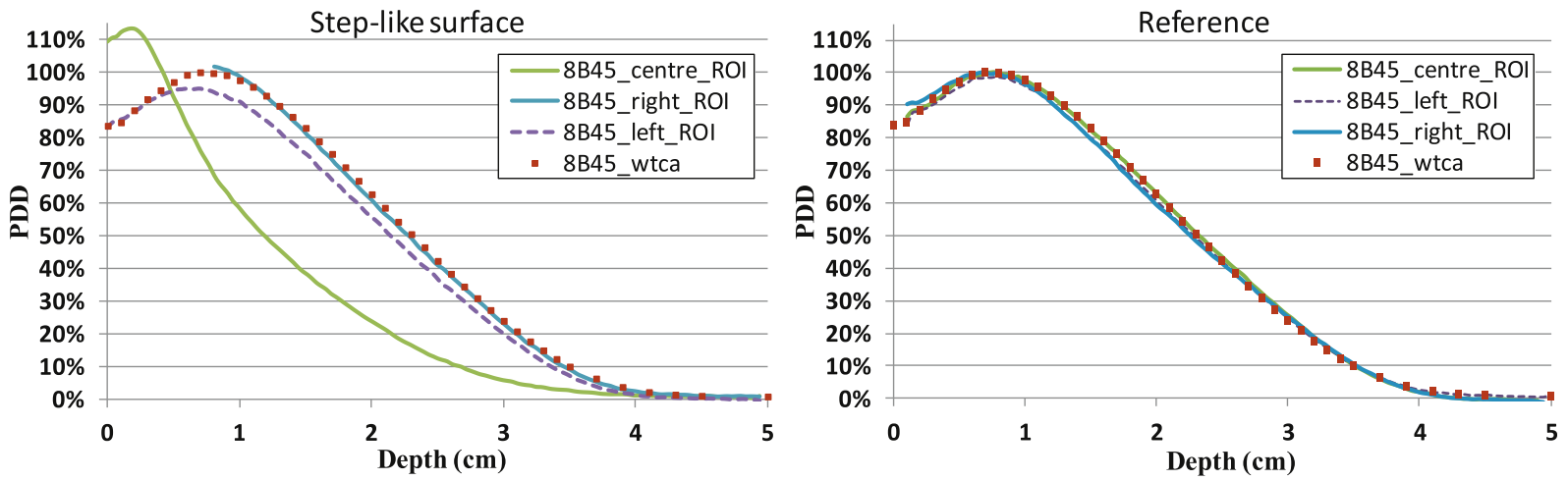

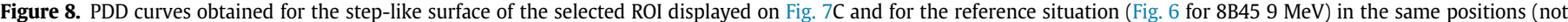

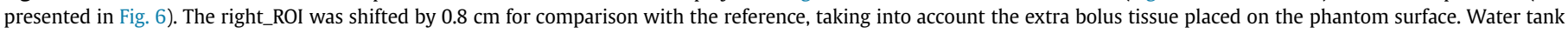
central axis (wtca) measurements are presented for comparison. 


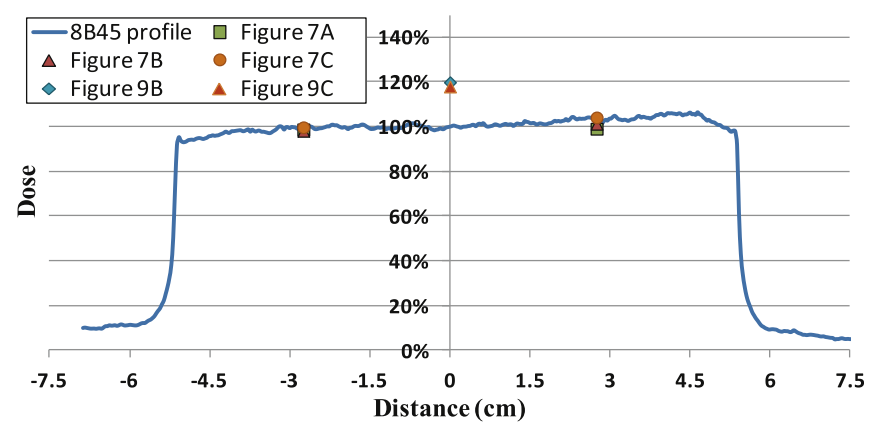

Figure 10. Central surface dose profile for $8 \mathrm{~B} 45$ applicator, $9 \mathrm{MeV}$, in reference conditions, and comparison with surface measurements with $1.5 \times 1.5 \mathrm{~cm}^{2}$ film pieces placed on the surface of the phantoms for non-reference conditions (step-like and curved surfaces).

results are affected by noise and spatial averaging. Considering all these sources of uncertainty, an agreement $\leq 3 \%$ confirms that absolute dose values obtained with the bolus set-up are sufficiently reliable to be used for normalization purposes.

Therefore, the bolus set-up was considered validated and used to obtain dose distributions for non-parallel and/or step-like (Fig. 7) and curved (Fig. 9) irradiation surfaces, with good visualization of the clinically relevant effects, but with a controlled geometry. All dose distributions were normalized to the value of dose maximum $\left(D_{\max }\right)$ in the central axis, obtained for the reference distributions shown in Fig. 6. This methodology allows easy comparison of results in terms of percentage, because the $100 \%$ curve corresponds to the same value of absolute dose, in reference and non-reference distributions obtained for the same applicator.

When the irradiated surface presents step-like irregularities (Fig. 7B and C), some noticeable effects occur: the dose distribution is shifted upwards under the extra tissue, as expected, but a hot spot is also generated near the step edge, not adjacent but slightly displaced sideways. This seems to result from a combination of lateral scatter with non-perpendicular irradiation. The PDD curve presented in Fig. 8, obtained from the central ROI in Fig. 7C, provides a more quantitative analysis, showing that the hot spot corresponds to a $13 \%$ increase of the maximum dose. The depth of dose maximum $\left(\mathrm{d}_{\max }\right)$ is also shifted towards the surface, with a quick decrease of dose beyond this depth.

In the same irradiation geometry (Fig. 7C), there is an air gap of $0.8 \mathrm{~cm}$ between the surface and the applicator on the left side, resulting in a lower value of $D_{\max }$ in the PDD curve shown in Fig. 8 (left_ROI) when compared with the reference, despite similar dose values near the surface. The PDD curve from the right_ROI, taken below the bolus, is almost coincident with the PDD curve obtained on the right_ROI in the reference, when shifted by $0.8 \mathrm{~cm}$ in depth, which is the thickness of the bolus when compressed between the applicator and the solid water phantom. The surface measurements presented in Fig. 10 for this irradiation geometry (Fig. 7C) are approximately the same as expected for the reference. This result is in good agreement with the left_ROI and right_ROI PDD curves shown in Fig. 8, despite slightly different locations.

The results presented in Fig. 7 also show the effects of air gaps in combination with non-parallel alignment on the dose distribution. When the gantry angle is $40^{\circ}$, with and without bolus (Fig. 7A and B), surface measurements show a small decrease in dose, particularly on the right side of the profile (Fig. 10). Since this effect was not observed for the parallel geometry of Fig. 7C, it is probably due to the altered gantry angle, rather than the air gap. The behaviour observed in Fig. 7B, for a step-like surface and non-parallel irradiation, is similar to that seen in Fig. $7 \mathrm{C}$, but includes also the effects seen in Fig. 7A as a result of the non-parallel alignment of the applicator. The profile is not as shallow as in Fig. 7C, but the hotspot is still there.

The dose distributions resulting from curved irradiation surfaces are presented in Fig. 9. The situations presented in Fig. 9A and B are the most clinically realistic scenarios. The one created in Fig. 9B is unlikely, because the applicator is too long. But if the choice of the applicator is dictated by width and inclination of the surface to be irradiated, the length may not be a perfect fit.

In Fig. 9A, the applicator (6B45) is close to the borders of the concavity. Comparing this distribution with the reference presented in Fig. 6 for a 6B45, it appears to be shifted forward, becoming curved and longer. This could be a problem for target coverage, since the prescription in IOERT is usually done to the $90 \%$ isodose. The effect is also visible for the other two irradiation geometries (Fig. 9B and C), but additional effects are observed due to the bolus covering part of the applicator entrance. It is interesting to note that the hotspot created by the presence of extra tissue in the step-like surfaces is still visible in Fig. 9B and C, even though this time there is no step-like edge, but only a slope. The hotspot followed by a quick reduction of dose is similar to the effect observed in Fig. 7B and C, although the decrease is even quicker for curved surfaces. These effects confirm the importance of obtaining 2D distributions resulting from clinically relevant irradiation surfaces, and providing this information to the radiation oncologists.

The $1.5 \times 1.5 \mathrm{~cm}^{2}$ film pieces placed at the centre of the curved surfaces registered higher dose readings $(+19$ and $+21 \%$ respectively) when compared with the reference situation, as shown in Fig. 10. These results are consistent with the dose distributions presented in Fig. 9B and C, where the presence of a hotspot is clearly visible. In addition, this dose increase at the surface is similar to the results obtained at the centre_ROI in Fig. 8 for the step-like parallel irradiation of Fig. $7 \mathrm{C}$, where an increase of the surface dose of more than $20 \%$ was observed. However, in this case, the surface dose was influenced by an asymmetry in two directions, because the curved surface surrounds the film piece, as shown in Fig. $1 \mathrm{G}$ and $\mathrm{H}$, and the distance to the curved slope is shorter in the direction perpendicular to the dose distribution of Fig. 9.

For the 6B45 irradiation, the surface dose measured at the isocentre was $5 \%$ lower than expected in the reference situation. This is in good agreement with the dose distribution of Fig. 9A, where no hotspot is observed, probably because the applicator opening was the same size as the curved surface.

Slab phantoms and Gafchromic EBT3 films are easy to use and require less machine and irradiation time than water tank measurements. This is very important for IOERT, because machine time is always scarce with conventional LINACs, and there is a large number of applicator diameters and bevels to characterize. It is also important for mobile LINACs which, for security reasons, need to be moved to a shielded bunker for measurements requiring large irradiation doses [6]. The results presented here are for a conventional LINAC adapted with a hard-docking IOERT system. Mobile LINACs have flatter dose profiles, and PDD curves with higher surface doses [6], although electron energies and applicator shape are similar, this may lead to different results for similarly shaped surfaces.

Solid water phantoms proved a powerful and effective means to obtain quick visualization of dose distributions in relevant situations. However, the use of solid phantoms has its limitations, and Monte Carlo computational methods will probably be necessary to create systematic simulations of multiple scenarios, obtain complete 3D distributions, and to quantify the influence of different parameters. Such a large-scale study falls outside the scope of this work. 


\section{Conclusion}

The bolus set-up shown in Fig. 1D proved a practical and easy way to obtain detailed IOERT dose distributions in the direction parallel to the incident beam using Gafchromic EBT3 films, which can be converted into colour coded graphical representations for easier visualization of isodoses. Gamma function analysis with $2 \%$ and $2 \mathrm{~mm}$ criteria showed good agreement between the dose distributions obtained with this setup, and those obtained in a water phantom with a diode detector, between $2 \mathrm{~mm}$ below the surface and R10, for all applicators and beam energies studied.

This set-up was used to assess the effect of step-like and curved irradiation surfaces. The results obtained confirm that the shape of the surface may have a considerable effect on the dose distribution. When the applicator is partly covered by tissue, adjacent hotspots are generated close to the surface, followed by a quick decrease of dose at underlying depth. Curved irradiation surfaces cause the dose distributions to be shifted forward and to greater depth.

These results highlight the importance of understanding in more detail the dose distributions resulting from irregular surfaces, both for optimization purposes as well as to interpret in vivo measurements in pelvic IOERT. Phantom measurements can be used to obtain dose distributions for a few select situations, for visualization by radiation oncologists.

\section{Acknowledgements}

The authors are grateful to L. Cunha, A. Dias, A. Esposito, J. Lencart and S. Silva for useful discussions, to P. Limede for help with measurements of sacrum dimensions, and to all the Radiotherapy Technologists working at $2100 \mathrm{CD}$, for their collaboration with these measurements.

This work was supported by national funds through Fundação para a Ciência e Tecnologia - FCT, in the framework of the project PTDC/SAU-ENB/117631/2010, which is cofinanced by FEDER, through Programa Operacional Fatores de Competitividade COMPETE of QREN (reference FCOMP-01-0124-FEDER-021141).

\section{References}

[1] Calvo F, Meirino RM, Orecchia R. Intraoperative radiation therapy first part: rationale and techniques. Crit Rev Oncol Hematol 2006;59:106-15. http:// dx.doi.org/10.1016/j.critrevonc.2005.11.004.

[2] Palta JR, Biggs PJ, Hazle JD, Huq MS, Dahl RA, Ochran TG, et al. Intraoperative electron beam radiation therapy: technique,dosimetry, and dose specification: report of task force 48 of the radiation therapy committee, american association of physicists in medicine. Int J Radiat Oncol Biol Phys 1995;33:725-46.

[3] Calvo F, Sole C, Herranz R, Lopez-Bote M, Pascau J, Santos A, et al. Intraoperative radiotherapy with electrons: fundamentals, results, and innovation Ecancer 2013;7:339. http://dx.doi.org/10.3332/ecancer.2013.339.

[4] Pascau J, Ph D, Santos A, Morillo V, Calvo FA, Bouche A, et al. An innovative tool for intraoperative electron beam radiotherapy simulation and Planning: description and initial evaluation by radiation oncologists. Int J Radiat Oncol Biol Phys 2012;83:287-95. http://dx.doi.org/10.1016/j.ijrobp.2011.12.063.

[5] Del Río J, Lázaro RA, Rojas RJ, Cores SG, Ruiz CG, Jesús M, et al. Descripción técnica y dosimétrica de un sistema aplicador de alineación rígida para radioterapia intraoperatoria con haces de electrones en acelerador convencional technical and dosimetric description of a hard-docking applicator system for intraoperative. Rev Fis Med 2013:4:131-42.

[6] Beddar AS, Biggs PJ, Chang S, Ezzell GA, Faddegon BA, Hensley FW, et al. Intraoperative radiation therapy using mobile electron linear accelerators: report of AAPM radiation therapy Committee task Group No. 72. Med Phys 2006:33:1476-89.

[7] Consorti R, Petrucci A, Fortunato F, Soriani A, Marzi S, Iaccarino G, et al. In vivo dosimetry with MOSFETs: dosimetric characterization and first clinical results in intraoperative radiotherapy. Int J Radiat Oncol Biol Phys 2005;63:952-60. http://dx.doi.org/10.1016/j.ijrobp.2005.02.049.

[8] Agostinelli S, Gusinu M, Cavagnetto F, Garelli S, Zeverino M, Guenzi M, et al. On-line optimization of intraoperative electron beam radiotherapy of the breast. Radiother Oncol 2012;103:188-92. http://dx.doi.org/10.1016 j.radonc.2012.01.009.
[9] Oshima T, Aoyama Y, Shimozato T, Sawaki M, Imai T, Ito Y, et al. An experimental attenuation plate to improve the dose distribution in intraoperative electron beam radiotherapy for breast cancer. Phys Med Biol 2009;54: 3491-500. http://dx.doi.org/10.1088/0031-9155/54/11/014.

[10] Soriani A, Iaccarino G, Felici G, Ciccotelli A, Pinnaro P, Giordano C, et al. Development and optimization of a beam shaper device for a mobile dedicated IOERT accelerator. Med Phys 2012;39:6080-9. http://dx.doi.org/ 10.1118/1.4749968.

[11] Azinovic I, Martinez Monge R, Aristu JJ, Salgado E, Villafranca E, Hidalgo OF, et al. Intraoperative radiotherapy electron boost followed by moderate doses of external beam radiotherapy in resected soft-tissue sarcoma of the extremities. Radiother Oncol 2003;67:331-7. http://dx.doi.org/10.1016/S01678140(03)00163-4.

[12] Krengli M, Calvo F a, Sedlmayer F, Sole CV, Fastner G, Alessandro M, et al. Clinical and technical characteristics of intraoperative radiotherapy. Analysis of the ISIORT-Europe database. Strahlenther Und Onkol 2013;189:729-37. http://dx.doi.org/10.1007/s00066-013-0395-1.

[13] Julián M de la C, Murillo EC, Gutiérrez FC, Ruiz-Zorrilla JG, díaz Fuentes R, Sánchez AF, et al. Reducción del número de medidas necesarias para la caracterización de un sistema de aplicadores de radioterapia intraoperatoria reduction in the number of measurements needed in order to characterize a system of intraoperative radiotherapy applicators. Rev Fis Med 2011;12: 97-102.

[14] Beddar a S, Briere TM, Ouzidane M. Intraoperative radiation therapy using a mobile electron linear accelerator: field matching for large-field electron irradiation. Phys Med Biol 2006;51:N331-7. http://dx.doi.org/10.1088/00319155/51/18/N01.

[15] Moylan R, Aland T, Kairn T. Dosimetric accuracy of Gafchromic EBT2 and EBT3 film for in vivo dosimetry. Australas Phys Eng Sci Med 2013;36:331-7. http:// dx.doi.org/10.1007/s13246-013-0206-0.

[16] Su F-C, Liu Y, Stathakis S, Shi C, Esquivel C, Papanikolaou N. Dosimetry characteristics of GAFCHROMIC EBT film responding to therapeutic electron beams. Appl Radiat Isot 2007;65:1187-92. http://dx.doi.org/10.1016/ j.apradiso.2007.05.005.

[17] Sorriaux J, Kacperek A, Rossomme S, Lee J a, Bertrand D, Vynckier S, et al. Evaluation of Gafchromic ${ }^{\circledR}$ EBT3 films characteristics in therapy photon, electron and proton beams. Phys Medica 2013;29:599-606. http://dx.doi.org/ 10.1016/j.ejmp.2012.10.001.

[18] Doppke KP, Hogstrom KR, Kutcher GJ, Nath R, Prasad SC, Purdy JA, et al. Clinical electron-beam dosimetry: report of AAPM radiation therapy committee task group No. 25. 1991.

[19] El Barouky J, Fournier-Bidoz N, Mazal A, Fares G, Rosenwald J-C. Practical use of Gafchromic(®) EBT films in electron beams for in-phantom dose distribution measurements and monitor units verification. Phys Med 2011;27:81-8. http://dx.doi.org/10.1016/j.ejmp.2010.04.001.

[20] Catalano M, Agosteo S, Moretti R, Andreoli S. Montecarlo simulation code in optimisation of the intraOperative radiation therapy treatment with mobile dedicated accelerator. J Phys Conf Ser 2007;74:0-11. http://dx.doi.org/ 10.1088/1742-6596/74/1/012002.

[21] Kesen ND, Cakir A, Okutan M, Bilge H. Research of dosimetry parameters in small electron beams. Sci Technol Nucl Install 2014;2014:1-7. http:// dx.doi.org/10.1155/2014/585219.

[22] Bufacchi A, Carosia A, Adorantea N, delle Cannea S, Malatestaa T, Capparellaa R, Fragomenia R, Bonannib A, Leoneb M, Marmirolib LB L. In vivo EBT radiochromic film dosimetry of electron beam for total skin electron therapy (TSET). Phys Medica 2007;23:67-72.

[23] Ciocca M, Orecchia R, Garibaldi C, Rondi E, Luini A, Gatti G, et al. In vivo dosimetry using radiochromic films during intraoperative electron beam radiation therapy in early-stage breast cancer. Radiother Oncol 2003;69:285-9. http://dx.doi.org/10.1016/j.radonc.2003.09.001.

[24] Severgnini M, De Denaro M, Bortul M, Vidali C, Beorchia A. In vivo dosimetry and shielding disk alignment verification by EBT3 GAFCHROMIC film in breast IOERT treatment. J Appl Clin Med Phys 2015;16:112-20.

[25] Ciocca M, Piazzi V, Lazzari R, Vavassori A, Luini A, Veronesi P, et al. Real-time in vivo dosimetry using micro-MOSFET detectors during intraoperative electron beam radiation therapy in early-stage breast cancer. Radiother Oncol 2006;78:213-6. http://dx.doi.org/10.1016/j.radonc.2005.11.011.

[26] I.A.E.A.. An International code of practice for dosimetry based on absorbed dose to water, series No.398, Absorbed dose determination in external beam radiotherapy. 2000.

[27] Borcia C, Mihailescu D. Are water-equivalent materials used in electron beams dosimetry really water equivalent? Rom Journ Phys 2008;53:851-63.

[28] Tamponi M, Bona R, Poggiu A, Marini P. A practical tool to evaluate dose distributions using radiochromic film in radiation oncology. Phys Medica 2014;31:31-6. http://dx.doi.org/10.1016/j.ejmp.2014.07.009.

[29] Girard F, Bouchard H, Lacroix F. Reference dosimetry using radiochromic film. J Appl Clin Med Phys 2014;13:1-12.

[30] Low DA, Harms WB, Mutic S, Purdy JA. A technique for the quantitative evaluation of dose distributions. Med Phys 1998;25:656-61.

[31] Devic S. Radiochromic film dosimetry: past, present, and future. Phys Med 2011;27:122-34. http://dx.doi.org/10.1016/j.ejmp.2010.10.001. 